$\$$ Research Square
Preprints are preliminary reports that have not undergone peer review.

They should not be considered conclusive, used to inform clinical practice, or referenced by the media as validated information.

\title{
Lymphocyte-C reactive protein ratio is a promising indicator for predicting intestinal necrosis in neonates with portal venous gas
}

Jian Cao

Children's Hospital of Chongqing Medical University

Xinyin Zhang

Children's Hospital of Chongqing Medical University

Jinfeng Hou

Children's Hospital of Chongqing Medical University

Mengying Cui

Children's Hospital of Chongqing Medical University

Pengfei Chen

Children's Hospital of Chongqing Medical University

\section{Qingshuang Liu}

Children's Hospital of Chongqing Medical University

Chunbao Guo

Children's Hospital of Chongqing Medical University

\section{Zhenhua Guo}

Children's Hospital of Chongqing Medical University

Yi Wang

Children's Hospital of Chongqing Medical University

Wei Liu (D3634158607@qq.com )

Children's Hospital of Chongqing Medical University

\section{Research Article}

Keywords: Portal venous gas, Neonate, C-reactive protein, Lymphocyte, Intestinal necrosis, Surgical timing

Posted Date: March 2nd, 2022

DOI: https://doi.org/10.21203/rs.3.rs-1341309/v1

License: (1) (1) This work is licensed under a Creative Commons Attribution 4.0 International License. Read Full License 


\section{Abstract}

Purpose: Portal venous gas (PVG) in neonates is a special abdominal imaging, which often indicates the occurrence of intestinal ischemia and necrosis, and is also an indicator to evaluate the severity and surgical timing of neonatal necrotizing enterocolitis. The timely identification and intervention of intestinal necrosis in neonates with PVG is particularly important, but it is still difficult at present. Various inflammatory factors in predicting prognosis and surgery timing have been described in adult literature, but rarely in neonatal literature. Therefore, we investigate the value of inflammatory factors in predicting intestinal necrosis in neonates with PVG.

Methods: We retrospectively reviewed the medical records of neonates with PVG detected by ultrasound in a tertiary-level referral hospital from January 2020 to December 2020. During the study period, 168 neonates with ultrasonographically identified PVG were included and were divided into two groups according to the presence of intestinal necrosis: a necrotic group $(n=35)$ and a nonnecrotic group $(n=133)$. We evaluated the predictive values of various combination of inflammatory markers in preoperative period laboratory analyses using the receiver operating characteristic (ROC) method.

Results: In the current cohort, a total of 168 patients were identified. Of these, 35 patients $(20.8 \%)$ underwent intestinal resection due to intestinal necrosis and 5 patients $(3.0 \%)$ developed intestinal stricture after medical management. The overall survival rate was $164 / 168$ (97.6\%). In patients with intestinal necrosis, platelet count $(\mathrm{p}<0.001)$, lymphocyte count $(\mathrm{p}<0.001)$, Eosinophil count $(p<0.001), C R P$ (C-reactive protein, $p<0.001)$, PLR (platelet-lymphocyte ratio, $p=0.002)$, NLR (neutrophil-lymphocyte ratio, $p=0.001$ ), LCR (lymphocyte-CRP ratio, $p<0.001$ ) and PCR (platelet- CRP ratio, $p<0.001$ ) values were significantly different with those in the patients without intestinal necrosis. Receiver operating characteristic (ROC) analysis results showed that the combination of C-reactive protein levels along with lymphocyte count (LCR) had the highest correlation with intestinal necrosis in neonates with portal venous gas [AUC $0.86(95 \% \mathrm{Cl} 0.79-0.94) ; \mathrm{p}<0.001$ ], with sensitivity of $0.81(0.73-0.87)$ and specificity of $0.86(0.69-0.95)$ for the diagnosis of intestinal necrosis.

Conclusions: The preoperative LCR score is a promising indicator for predicting intestinal necrosis in neonates with PVG, which could be used as an additional criterion to guide surgical management.

\section{Introduction}

Portal venous gas (PVG), which is defined as the presence of gas in the portal venous system, is a rare radiologic sign first recognized in neonates with necrotizing enterocolitis (NEC) in 1955 by Wolfe[1]. This finding has historically been considered a poor prognosis and life-threatening factor in neonates with NEC for a long time, as it represents a late sign of intestinal ischemia and a relative indicator for surgical intervention[2-4]. With the development of radiology, more and more recent literatures have reported that PVG is not only present in neonates with intestinal ischemia, but also in those with benign condition, such as bowel inflammation, food allergies and so on[5-7]. Moreover, the strategy of PVG alone as an indication for surgery has been questioned and challenged[8-10]. Therefore, considering the life-threatening impact of intestinal necrosis on neonates, it is particularly important to identify new validated predictors for surgical intervention in neonates with PVG. However, few studies have reported about the surgical indications and prognosis in neonates with PVG.

The role of systemic inflammatory markers in predicting surgical outcomes and oncological prognosis has been widely described in adult studies, but rarely in neonatal studies[11-13]. Recently, several studies have shown that the systemic inflammatory markers such as CRP (C-reactive protein), CAR (C-reactive protein-albumin ratio) and LCR (Lymphocyte-C reactive protein ratio) are recognized as the predictors of intestinal necrosis[14-15]. Based on this understood, some systemic inflammatory markers may predict the occurrence of intestinal necrosis in neonates with PVG.

The aim of this study was to describe the characteristics and outcomes of a recent cohort of neonates with PVG at a tertiary-level referral hospital, and to investigate whether the systemic inflammatory markers have the potential to predict intestinal necrosis in neonates with PVG.

\section{Materials And Methods}




\subsection{Study population and design}

A retrospective cohort study of all neonates with PVG diagnosed by ultrasonography using a Philips iU 22 or a Aixplorer machine that were consecutively admitted to the specialized neonatal intensive care unit at the Chongqing Children's Hospital (i.e. large, tertiary- level referral hospital) from 1 January 2020 to 31 December 2020 was performed. All ultrasound images of patients were reviewed on the picture archiving and communication systems monitor (PACS) by the experienced attending ultrasound diagnostic physicians and the observations were noted by consensus. Inclusion criteria was ultrasound imaging evidence of PVG which was Confirmed by two experienced ultrasound diagnostic physicians. Exclusion criteria were severe cardiopulmonary malformation resulting in rapid death of the neonate due to cardiopulmonary failure and significant intestinal perforation. Finally, a total of 168 neonates with PVG were included in current study and were divided into two groups according to the presence of intestinal necrosis: a necrotic group and a non-necrotic group. The necrotic group was defined as the group undergoing surgery due to intestinal necrosis. The non-necrotic group was defined as the group that did not undergo surgery. The presence of intestinal necrosis was comprehensively confirmed by pathological reports and surgical findings.

\subsection{Data assessment}

The demographic characteristics of neonates and mothers were collected from the electronic medical records: gestational age (GA), birth weight (BW), gender, maternal age, mode of delivery and multiple pregnancies. Simultaneously, the principal clinical manifestations, clinical radiographic and complications were collected too. In addition, inflammation associated biomarkers at the time of diagnosis of PVG such as platelets, lymphocytes, neutrophils, eosinophils, CRP, NLR, PLR, LCR, PCR were further measured and analyzed to identify the highest accuracy one for prediction intestinal necrosis in neonates with PVG.

\subsection{Statistics}

The statistical analyses were performed using software SPSS (Version 22.0, SPSS Inc., Chicago, IL). The continuous data were expressed as the mean \pm SD (standard deviation) or the median and interquartile range (IQR) and compared using t-tests or MannWhitney $U$ test according to data distribution. The categories variables were expressed as number (\%) and analyzed by Chi-squared test or Fisher's exact test, when applicable. A Receiver operating characteristic curve (ROC curve) was generated to value the various parameters for predicting intestinal necrosis through the area under the curve (AUC) calculation and to assess optimal cut-off points for the different parameters. The results with $\mathrm{P}<0.05$ were considered statistically significant.

\subsection{Ethics Committee Approval}

The current study protocol was performed following the expedited ethical committee approval by the Institutional Review Board of the Chongqing Medical University under the protection of personal information and was registered under number CHMU2021-189.

\section{Results}

\subsection{Patients' baseline characteristics}

A total of 168 consecutive neonates with confirmed PVG were identified during the study period and were subjected to final investigation. Of these, 87 patients (51.8\%) were diagnosed with necrotizing enterocolitis (NEC), 35 patients (20.8\%) with NEC were managed with intestinal resection due to bowel necrosis and 5 patients (3.0\%) developed intestinal stricture after medical management. The mortality rate of neonates with PVG in our study was only $0.02 \%(4 / 168)$. The baseline features of the neonates with PVG among two groups were presented in Table 1. As shown in Table 1, the demographic characteristics were comparable, including gestational age, birth weight, mode of delivery. The neonates suffering from bowel necrosis were more likely lower gestational age $(P<0.001)$ and lower birth weight $(P<0.001)$. In addition, there were more presentations of abdominal distension and vomiting in the neonates with intestinal necrosis $(\mathrm{P}<0.001)$. No statistic differences were detected in terms of gender and age at diagnosis between the two groups.

Table 1

Baseline characteristic of 168 neonates with PVG 


\begin{tabular}{|c|c|c|c|}
\hline & $\begin{array}{l}\text { Necrotic group } \\
(n=35)\end{array}$ & $\begin{array}{l}\text { Non-necrotic group } \\
(n=133)\end{array}$ & $P=$ \\
\hline Age at diagnosis $\llbracket \mathrm{d} \rrbracket$ & 10(7-19) & 11(7-16) & $P=0.581$ \\
\hline Weight at diagnosis $₫ \mathbf{g} \rrbracket$ & 2000ه1780-2290》 & $2830 \rrbracket 2375-3440 \rrbracket$ & $P<0.001$ \\
\hline Gender & & & $P=0.205$ \\
\hline Male & $15 \rrbracket 42.9 \% \rrbracket$ & $73 \llbracket 54.9 \% \rrbracket$ & \\
\hline Female & $20 \bigotimes 57.1 \% \rrbracket$ & $60 \rrbracket 45.1 \% \rrbracket$ & \\
\hline Gestational age & & & $P<0.001$ \\
\hline Term & $3 \rrbracket 8.6 \% \rrbracket$ & $81 \rrbracket 60.9 \% \rrbracket$ & \\
\hline Preterm $₫ \leq 37$ weeks $\rrbracket$ & $30 \otimes 85.7 \% \rrbracket$ & $51 \rrbracket 38.3 \% \rrbracket$ & \\
\hline Very preterm $₫ \leq 28$ weeks $\rrbracket$ & $2 \varangle 5.7 \% \rrbracket$ & $1 \rrbracket 0.8 \% \square$ & \\
\hline Birth weight & & & $P<0.001$ \\
\hline Normal & $3 \llbracket 8.6 \% \rrbracket$ & $91 \rrbracket 68.4 \% \rrbracket$ & \\
\hline Low birth weight $(\leq 2500 \mathrm{~g})$ & $23 \otimes 65.7 \% \rrbracket$ & $36 \rrbracket 27.1 \% \rrbracket$ & \\
\hline Very low birth weight $₫ \leq 1500 \mathrm{~g} \rrbracket$ & $9 \llbracket 25.7 \% \rrbracket$ & $6 \rrbracket 4.5 \% \rrbracket$ & \\
\hline Pregnancy & & & $P<0.001$ \\
\hline Single & $16 \rrbracket 45.7 \% \rrbracket$ & $110 \rrbracket 82.7 \% \rrbracket$ & \\
\hline Twin & $19 \otimes 54.3 \% \rrbracket$ & $23 \rrbracket 17.3 \% \square$ & \\
\hline Mode of delivery & & & $P=0.02$ \\
\hline Normal delivery & $2 \searrow 5.7 \% \rrbracket$ & $43 \rrbracket 32.3 \% \rrbracket$ & \\
\hline Cesarean delivery & $33 \otimes 94.3 \% \square$ & $90 \rrbracket 67.7 \% \rrbracket$ & \\
\hline \multicolumn{4}{|l|}{ Clinical symptoms } \\
\hline Fever & $8 \rrbracket 22.9 \% \rrbracket$ & $20 \rrbracket 15 \% \rrbracket$ & $P=0.269$ \\
\hline Diarrhea & $3 \rrbracket 8.6 \% \rrbracket$ & $40 \rrbracket 30.1 \% \rrbracket$ & $P=0.009$ \\
\hline Abdominal distension & $31 \rrbracket 88.6 \% \rrbracket$ & $46 \rrbracket 34.6 \% \rrbracket$ & $P<0.001$ \\
\hline Vomiting & $28 \llbracket 80 \% \rrbracket$ & $48 \bowtie 36.1 \% \square$ & $P<0.001$ \\
\hline Blood stool & $29 \bigotimes 82.9 \% \rrbracket$ & 106ه79.7\%区 & $P=0.676$ \\
\hline
\end{tabular}

Values are presented as median (IQR) or $n(\%)$

Abbreviations: IQR=interquartile range.

\subsection{Patients' laboratory parameters}

To identify the potential of laboratory parameters for predicting intestinal necrosis, we detected several hematologic parameters that may reflect intestinal inflammatory conditions, such as WBC (white blood cells), platelets, lymphocytes, neutrophils, eosinophils, and CRP (C-reactive protein). Further, we explored some combinations of above indicators, especially the up and down regulation combinations, including PLR (platelet-lymphocyte ratio), LCR (lymphocyte-CRP ratio), NLR (neutrophil-lymphocyte ratio), PCR (platelet- CRP ratio). The hematologic parameters of the neonates with PVG among two groups were presented in Table 2. As shown in Table 2, the intestinal necrosis was associated with the higher $\operatorname{CRP}(P<0.001), P L R(P=0.002)$ and NLR( $P=0.002)$, but with the lower WBC $(P=0.005)$, platelets $(P<0.001)$, lymphocytes $(P<0.001)$, eosinophils $(P<0.001), P C R(P<0.001)$ and $L C R(P<0.001)$. Moreover, the 
hemoglobin level in group with intestinal necrosis was significantly lower than that in group without intestinal necrosis $(P=0.011)$. No statistic difference was detected in neutrophil count between the two groups.

Table 2

Comparison of the hematologic parameters of the groups

\begin{tabular}{|llll|}
\hline & $\begin{array}{l}\text { Necrotic group } \\
(\mathrm{n}=35)\end{array}$ & $\begin{array}{l}\text { Non-necrotic group } \\
(\mathrm{n}=133)\end{array}$ & $\mathrm{P}=$ \\
\hline WBC(10/L) & $6.79(4.48-13.28)$ & $10.6(8.595-13.28)$ & $\mathrm{P}=0.005$ \\
\hline Hb level(g/dL) & $138.06 \pm 33.85$ & $152.19 \pm 27.53$ & $\mathrm{P}=0.011$ \\
\hline Platelets(109/L) & $228.00(185.00-299.00)$ & $338.00(267.50-432.50)$ & $\mathrm{P}<0.001$ \\
\hline Lymphocytes(10/L) & $1.75(1.19-3.33)$ & $4.42(3.00-5.61)$ & $\mathrm{P}<0.001$ \\
\hline Neutrophils(10/L) & $4.91(1.95-8.04)$ & $5.17(3.42-6.55)$ & $\mathrm{P}=0.459$ \\
\hline Eosinophils(10/L) & $0.00(0.00-0.10)$ & $0.28(0.11-0.51)$ & $\mathrm{P}<0.001$ \\
\hline CRP(g/L) & $18.00(5.00-38.00)$ & $5.00(3.00-7.00)$ & $\mathrm{P}<0.001$ \\
\hline PLR & $127.22(70.24-230.21)$ & $80.66(59.35-113.36)$ & $\mathrm{P}=0.002$ \\
\hline NLR & $2.44(1.04-4.59)$ & $1.10(0.76-1.80)$ & $\mathrm{P}=0.001$ \\
\hline LCR & $0.12(0.05-0.23)$ & $0.55(0.35-0.70)$ & $\mathrm{P}<0.001$ \\
\hline PCR & $12.50(7.14-25.00)$ & $33.33(25.00-50.00)$ & $\mathrm{P}<0.001$ \\
\hline
\end{tabular}

Values are presented as median (IQR) or $M \pm S D$ or $n(\%)$

Abbreviations: IQR=interquartile range; $W B C=W$ hite blood cells; $\mathrm{Hb}=$ hemoglobin; $\mathrm{CRP}=\mathrm{C}$-reactive protein; $\mathrm{PLR}=$ platelet-lymphocyte ratio; NLR=neutrophil-lymphocyte ratio; $L C R=$ lymphocyte $-\mathrm{CRP}$ ratio; $\mathrm{PCR}=$ platelet- $\mathrm{CRP}$ ratio.

\subsection{Predictive Effects of hematologic parameters $₫$}

A ROC curve analysis was performed to value all these hematologic parameters for predicting intestinal necrosis. The results are shown in Table 3. As shown in Table 3, the areas under the curve of eosinophils, PCR and LCR were more significant (AUC=0.82, $P<0.001 ; A U C=0.84, P<0.001 ; A U C=0.86, P<0.001)$. A serum LCR of $\leq 0.27$ at the time of diagnosis of $P V G$ had the highest predictive ability at sensitivity of $81 \%(95 \% \mathrm{Cl} 73.3-87.2)$ and specificity of $86 \%(95 \% \mathrm{Cl} 69.0-94.6)$ in predicting intestinal necrosis in neonates with PVG (Table 3, Fig.1).

Table 3

ROC curve results and sensitivity, specificity values 


\begin{tabular}{lllllllllll} 
& WBC & Hb level & Platelets & Lymphocytes & Eosinophils & CRP & PLR & LCR & NLR & PCR \\
\hline AUC & 0.65 & 0.63 & 0.73 & 0.78 & 0.82 & 0.77 & 0.67 & 0.86 & 0.68 & 0.84 \\
$(95 \%$ Cl) & $(0.52-$ & $(0.51-$ & $(0.64-$ & $(0.68-0.89)$ & $(0.73-$ & $(0.67-$ & $(0.56-$ & $(0.79-$ & $(0.58-$ & $(0.76-$ \\
& $0.78)$ & $0.74)$ & $0.83)$ & & $0.90)$ & $0.88)$ & $0.79)$ & $0.94)$ & $0.79)$ & $0.92)$ \\
\hline p values & 0.005 & 0.022 & $<0.001$ & $<0.001$ & $<0.001$ & $<0.001$ & 0.002 & $<0.001$ & 0.001 & $<0.001$ \\
\hline Cut-off & $<6.87$ & $<117.50$ & $<272.50$ & $<3.42$ & $<0.06$ & $>8.5$ & $>124.59$ & $<0.27$ & $>1.37$ & $<17.51$ \\
\hline Sensitivity & 0.93 & 0.90 & 0.75 & 0.69 & 0.89 & 0.63 & 0.54 & 0.81 & 0.74 & 0.93 \\
$(95 \%$ Cl) & $(0.86-$ & $(0.84-$ & $(0.67-$ & $(0.60-0.76)$ & $(0.81-$ & $(0.45-$ & $(0.37-$ & $(0.73-$ & $(0.53-$ & $(0.87-$ \\
& $0.96)$ & $0.94)$ & $0.82)$ & & $0.93)$ & $0.78)$ & $0.71)$ & $0.87)$ & $0.85)$ & $0.97)$ \\
\hline Specificity & 0.51 & 0.37 & 0.66 & 0.83 & 0.71 & 0.90 & 0.83 & 0.86 & 0.63 & 0.63 \\
$(95 \%$ Cl) & $(0.34-$ & $(0.22-$ & $(0.48-$ & $(0.66-0.93)$ & $(0.53-$ & $(0.83-$ & $(0.75-$ & $(0.69-$ & $(0.54-$ & $(0.45-$ \\
& $0.68)$ & $0.55)$ & $0.80)$ & & $0.85)$ & $0.94)$ & $0.89)$ & $0.95)$ & $0.71)$ & $0.78)$
\end{tabular}

Abbreviations: WBC=White blood cells; $\mathrm{Hb}=$ hemoglobin; $\mathrm{CRP}=\mathrm{C}$-reactive protein; $\mathrm{PLR}=$ platelet-lymphocyte ratio; NLR=neutrophillymphocyte ratio; $L C R=l y m p h o c y t e-C R P$ ratio; $P C R=$ platelet- $C R P$ ratio.

\section{Discussion}

In our study, the mean gestational age and birth weight of neonates with PVG in necrotic group were significantly lower than those in non-intestinal necrosis group, which might reflect the correlation between surgery, gestational age and birth weight. This finding was consistent with the characteristics of neonates with NEC [16,17], which might be due to the distribution of disease in this study that most of patients (51.8\%) were diagnosed with NEC. In addition, the surgical rate was $20.8 \%$ and the mortality rate was $0.02 \%$, which might reflect the findings of several previous adult studies showing that most of the patients with PVG were in benign conditions that did not require emergency surgery[8,18].

In spite of this, considering the life-threatening impact of intestinal necrosis on neonates, timely recognition and intervention of intestinal necrosis in neonates with PVG is particularly important for the management of this disease. Previous studies have shown that the magnitude of systemic inflammation is correlated with the severity of intestinal ischemia. Yildirim $\mathrm{M}$ and Zhou $\mathrm{H}$ et al reported that combinations of inflammatory factors such as lymphocytes, neutrophils and CRP released by the ischemic intestinal wall could predict the occurrence of intestinal ischemia [14,19]. Amir T. Mohd Amin et al mentioned that CRP combined with albumin could predict surgical intervention and mortality in neonates with necrotizing enterocolitis[15]. Mengnan Yu et al demonstrated that white blood cell count and platelet count were closely related to surgery and death in neonates with necrotizing enterocolitis[20]. So, in the current study, we explored the value of different inflammatory factors in predicting intestinal necrosis in neonates with PVG. To our knowledge, no study has been reported about this in neonates with PVG before.

In present study, we found that leukocyte, platelet, lymphocyte, eosinophil, CRP values in necrotic group were significantly different from those in non-necrotic group. The surrogate marker of the severity of the inflammatory response were significantly higher for CRP value but lower for leukocyte, platelet, lymphocyte counts in the patients with intestinal necrosis. This result implies that higher concentration of CRP and lower plasma concentrations of leukocyte, platelet, lymphocyte are closely related to intestinal necrosis. However, the ability to predict intestinal necrosis in neonates with PVG by the single use of WBC or PLT was not great, so we further explored the predictive potential of combinations of inflammatory factors, including PLR, NLR, PCR LCR.

The significance of the PLR, NLR and LCR has previously been reported in a variety of adult conditions. It has been shown that NLR, PLR and LCR are useful markers in predicting the prognosis of patients with lung cancer[21], hepatocellular Carcinoma[22], colorectal Cancer[23], kidney disease [24] and COVID-19[25]. The higher LCR value has also been identified to be an independent predictor of intestinal ischemia in the patients with hernia strangulation[14]. To date, no studies have analyzed the combinations of inflammatory factors in neonates with PVG, especially the PCR (platelet- CRP ratio) firstly introduced in our study. Our result with ROC analysis showed among all these parameters, eosinophils, PCR and LCR were good inflammatory parameter, strongly related with intestinal necrosis. Compared with eosinophils and PCR, LCR is a more reliable indicator of intestinal resection in neonates with PVG. The cut-off value of LCR is 0.27 , which means if the probability is less than or equal to 0.27 , surgery should be considered. 
We acknowledge that our study has limitations. First, it was a single-center retrospective study with a relatively limited number of patients. Second, the surgical decision for intestinal necrosis depended on the opinion of the individual pediatric surgeon, which was subjective and therefore variable. Therefore, a further multicenter prospective study with more patients is needed to validate the role of preoperative LCR in neonates with PVG. Finally, inflammatory biomarkers should only be used as an additional criterion to guide surgical management. The final decision of surgery should be made in conjunction with the clinical manifestations of the patients.

\section{Conclusion}

The current research suggests that preoperative LCR of $\leq 0.27$ is a promising predictive factor for intestinal necrosis in neonates with PVG, which can be used as an additional criterion to guide surgical management. To date, no other studies have specifically addressed this issue specifically in neonates with PVG.

\section{Declarations}

\section{Ethics approval and consent to participate}

All procedures performed in studies involving human participants were in accordance with the ethical standards of the institutional and/or national research committee and with the 1964 Helsinki declaration and its later amendments or comparable ethical standards. The current study protocol was performed following the expedited ethical committee approval by the Institutional Review Board of the Children's Hospital of Chongqing Medical University under the protection of personal information and was registered under number CHMU2021-189. The Institutional Review Board of the Children's Hospital of Chongqing Medical University approved informed consent waiver.

\section{Consent for publication}

Not applicable

\section{Availability of data and materials}

The datasets generated during and analyzed during the current study are not publicly available due to ongoing analysis in other directions but are available from the corresponding author on reasonable request.

\section{Competing interests}

The authors declare that the research was conducted in the absence of any commercial or financial relationships that could be construed as a potential conflict of interest.

\section{Funding}

Not applicable

\section{Authors' Contributions}

Jian Cao designed and analyzed the data and wrote the paper. Xinyin Zhang, Jinfeng Hou, Mengying Cui, Pengfei Chen and Qingshuang Liu collected the data and performed the statistical calculations. Chunbao Guo, Zhenhua Guo, Yi Wang, Wei Liu evaluated and modified the manuscript.

\section{Acknowledgments}


We thank Prof. Yun He for providing technical assistance and for insightful discussions during the preparation of the manuscript and all organizations that funded my research.

\section{References}

1. Wolfe JN, Evans WA. Gas in the portal veins of the liver in infants; a roentgenographic demonstration with postmortem anatomical correlation. Am J Roentgenol Radium Ther Nucl Med 1955; 74(3): 486-8. PMID: 13249015

2. Buras R, Guzzetta P, Avery G, Naulty C. Acidosis and hepatic portal venous gas: indications for surgery in necrotizing enterocolitis. Pediatrics 1986; 78(2): 273-7. PMID: 3737303

3. Wiesner W, Mortelé KJ, Glickman JN, Ji H, Ros PR. Pneumatosis intestinalis and portomesenteric venous gas in intestinal ischemia: correlation of CT findings with severity of ischemia and clinical outcome. AJR Am J Roentgenol 2001; 177(6): 131923. doi: 10.2214/ajr.177.6.1771319

4. Naguib N, Mekhail P, Gupta V, Naguib N, Masoud A. Portal venous gas and pneumatosis intestinalis; radiologic signs with wide range of significance in surgery. J Surg Educ 2012; 69(1): 47-51. doi: 10.1016/j.jsurg.2011.07.005

5. Altokhais TI. Portal gas in neonates; is it always surgical? A case report. World J Clin Cases 2020; 8(17): 3804-7. doi: 10.12998/wjcc.v8.i17.3804

6. Daneshmand A, Parys S, Rao S, Watanabe Y, Sieunarine K. Portal venous gas: different aetiologies and their respective outcomes. ANZ J Surg 2020; 90(5): 767-71. doi: 10.1111/ans.15795

7. Siddique Z, Thibodeau R, Jafroodifar A, Hanumaiah R. Pediatric milk protein allergy causing hepatic portal venous gas: Case report. Radiol Case Rep 2021; 16(2): 246-9. doi: 10.1016/j.radcr.2020.11.002

8. Nelson AL, Millington TM, Sahani D, et al. Hepatic portal venous gas: the ABCs of management. Arch Surg 2009; 144(6): 575-81; discussion 81. doi: 10.1001/archsurg.2009.88

9. Abboud B, El Hachem J, Yazbeck T, Doumit C. Hepatic portal venous gas: physiopathology, etiology, prognosis and treatment. World J Gastroenterol 2009; 15(29): 3585-90. doi: 10.3748/wjg.15.3585

10. Cuna AC, Reddy N, Robinson AL, Chan SS. Bowel ultrasound for predicting surgical management of necrotizing enterocolitis: a systematic review and meta-analysis. Pediatr Radiol 2018; 48(5): 658-66. doi: 10.1007/s00247-017-4056-x

11. Okugawa Y, Toiyama Y, Yamamoto A, et al. Lymphocyte-C-reactive Protein Ratio as Promising New Marker for Predicting Surgical and Oncological Outcomes in Colorectal Cancer. Ann Surg 2020; 272(2): 342-51. doi: 10.1097/SLA.0000000000003239

12. Okugawa Y, Toiyama Y, Fujikawa H, et al. Prognostic Potential of Lymphocyte-C-Reactive Protein Ratio in Patients with Rectal Cancer Receiving Preoperative Chemoradiotherapy. J Gastrointest Surg 2021; 25(2): 492-502.doi: 10.1007/s11605-019-04495-4

13. Yildirim M, Koca B. Lymphocyte C-reactive protein ratio: A new biomarker to predict early complications after gastrointestinal oncologic surgery. Cancer Biomark 2021; 31(4): 409-17. doi: 10.3233/CBM-210251

14. Yildirim M, Dasiran F, Angin YS, Okan I. Lymphocyte-C-reactive protein ratio: a putative predictive factor for intestinal ischemia in strangulated abdominal wall hernias. Hernia 2021; 25(3): 733-9.doi:10.1007/s10029-020-02174-x

15. Mohd Amin AT, Zaki RA, Friedmacher F, Sharif SP. C-reactive protein/albumin ratio is a prognostic indicator for predicting surgical intervention and mortality in neonates with necrotizing enterocolitis. Pediatr Surg Int 2021; 37(7): 881-

6.doi:10.1007/s00383-021-04879-1

16. Rich BS, Dolgin SE. Necrotizing Enterocolitis. Pediatr Rev 2017; 38(12): 552-9. doi: 10.1542/pir.2017-0002

17. Knell J, Han SM, Jaksic T, Modi BP. Current Status of Necrotizing Enterocolitis. Curr Probl Surg 2019; 56(1): 11-38. doi: 10.1067/j.cpsurg.2018.11.005

18. Iannitti DA, Gregg SC, Mayo-Smith WW, Tomolonis RJ, Cioffi WG, Pricolo VE. Portal venous gas detected by computed tomography: is surgery imperative? Dig Surg 2003; 20(4): 306-15.doi: 10.1159/000071756

19. Zhou H, Ruan X, Shao X, Huang X, Fang G, Zheng X. Clinical value of the neutrophil/lymphocyte ratio in diagnosing adult strangulated inguinal hernia. Int J Surg 2016; 36(Pt A): 76-80.doi: 10.1016/j.ijsu.2016.10.026

20. Yu M, Liu G, Feng Z, Huang L. Combination of plasma white blood cell count, platelet count and C-reactive protein level for identifying surgical necrotizing enterocolitis in preterm infants without pneumoperitoneum. Pediatr Surg Int 2018; 34(9): 945- 
50. doi: 10.1007/s00383-018-4305-6

21. Mandaliya H, Jones M, Oldmeadow C, Nordman, II. Prognostic biomarkers in stage IV non-small cell lung cancer (NSCLC): neutrophil to lymphocyte ratio (NLR), lymphocyte to monocyte ratio (LMR), platelet to lymphocyte ratio (PLR) and advanced lung cancer inflammation index (ALI). Transl Lung Cancer Res 2019; 8(6): 886-94. doi: 10.21037/tlcr.2019.11.16

22. Yugawa K, Maeda T, Kinjo N, et al. Prognostic Impact of Lymphocyte-C-Reactive Protein Ratio in Patients Who Underwent Surgical Resection for Hepatocellular Carcinoma. J Gastrointest Surg 2021.doi:10.1007/s11605-021-05085-z

23. Stojkovic Lalosevic M, Pavlovic Markovic A, Stankovic S, et al. Combined Diagnostic Efficacy of Neutrophil-to-Lymphocyte Ratio (NLR), Platelet-to-Lymphocyte Ratio (PLR), and Mean Platelet Volume (MPV) as Biomarkers of Systemic Inflammation in the Diagnosis of Colorectal Cancer. Dis Markers 2019; 2019: 6036979.doi:10.1155/2019/6036979

24. Qin B, Ma N, Tang Q, et al. Neutrophil to lymphocyte ratio (NLR) and platelet to lymphocyte ratio (PLR) were useful markers in assessment of inflammatory response and disease activity in SLE patients. Mod Rheumatol 2016; 26(3): 3726.doi:10.3109/14397595.2015.1091136

25. Chan AS, Rout A. Use of Neutrophil-to-Lymphocyte and Platelet-to-Lymphocyte Ratios in COVID-19. J Clin Med Res 2020; 12(7): 448-53. doi: 10.3109/14397595.2015.1091136 